University of Birmingham, UK

A.McNally.1@bham.ac.uk Cite this as: BMJ 2021;372:n504 http://dx.doi.org/10.1136/bmi.n504 Published: 22 February 2021

\section{What makes new variants of SARS-CoV-2 concerning is not where they come from, but the mutations they contain}

\author{
What do the new SARS-CoV-2 mutations mean, and how should we track them?
}

\section{Alan McNally professor in microbial genomics}

In the past three months global attention has turned to the discovery and health risks of new variants of the SARS-CoV-2 virus. A new covid variant, B1525, has very recently been identified in the UK. In December, the discovery of what has become popularly known as the Kent variant, or B.1.1.7, saw the virus sweep across the UK and dominating infections because of an increase in transmissibility. This was followed by the South African variant B.1.351 which contains a mutation now being reported to reduce the efficacy of the ChAdOx vaccine to the extent that South Africa is removing it from itsvaccination programme. The Brazil variant P.1 is also spreading to multiple countries with worries about its propensity for causing reinfections, given its emergence in a place that may have already hit herd immunity in the first wave.

The world now seems obsessed with SARS-CoV-2 variants and designating them a place of origin. This is an unfortunate stigma that should be avoided, given that where a virus is first detected is not necessarily where it originated. Lest we forget, Spanish flu (the 1918 influenza pandemic) had no connection to Spain.

The fascination with variants is now becoming as contagious as the virus, and is provoking similar knee jerk reactions. The discovery of the B.1.351 variant in the UK has led to a mass scale targeted testing and sequencing regimen in affected areas in an attempt to control its spread. This is because of a press release that stated the ChAdOx vaccine did not protect against mild and moderate covid-19 symptoms in a small South African study of young adults. While prudence and quick action should always be advised in matters of covid, it's also worth remembering that none of the data is published and so a significant supposition is being made on the ability of the Oxford AstraZeneca vaccine to control B.1.351 cases. Borders are now being closed in efforts to prevent introductions of new variants of concern into the UK and other countries, but will this truly protect us?

What makes the variants concerning is not where they come from, but the mutations they contain. The B.1.1.7 virus is characterised by a deletion in the spike protein and a mutation at $\mathrm{N}_{501} \mathrm{Y}$ which enhances its transmissibility, as well as a potentially important mutation in the furin cleavage site. These mutations are found on a background of an unusually high number of other mutations, making B.1.1.7 distinct. In the case of B.1.351, what makes it a threat to vaccine efficacy is the E484K mutation in the spike protein, also seen in P.1. It is naïve, however, to think that these mutations of concern are restricted to singular geographically defined viral lineages.
What has driven the emergence of these phenotypically important mutations are significant selection pressures. In the case of B.1.1.7 random mutations conferring an increase in transmissibility led to an increase in fitness of the virus which was rapidly selected for and became dominant in the UK. In the case of B.1.351 it may be that selection is for a random mutation allowing some form of enhanced escape from immune pressure and onward transmission, generating a fitness advantage-although evidence remains weak for this. The key point here is that these selection pressures are not geographically specific. The virus is encountering similar selection pressures wherever it is transmitting and has relatively high prevalence, meaning that the selection for random mutations that can confer a fitness advantage can happen anywhere, and at any time. This is now being seen with the emergence of the E484K mutation in the B.1.1.7 virus variant, meaning that the Kent variant now has the important South Africa mutation.

The evidence of multiple introductions of SARS-CoV-2 into the UK over the course of 2020 is compelling enough to lead to a much stricter policy of border control. Indeed, this is a measure that should already have been in place in 2020. However, the need for near real time testing and genomic surveillance goes way beyond introductions from travel or targeting areas where B.1.351 has been found.

The speed and success of the UK vaccine policy means that new and strong selection pressures are being imposed on SARS-CoV-2 across the country. It is possible, maybe even likely, that this will lead to selection of new mutations and the emergence of new variants within this country. What we need now is the ability to match our testing capability with a rapid genoptyping or sequencing capability, with some form of genotyping applied in real time to positive samples. This would allow the kind of real time epidemiology that serendipitously occurred with B.1.1.7 because of the effect on the $S$ gene target amplification of the Thermo Fisher polymerase chain reaction assay used in many of the pillar 2 testing laboratories. The closer this genotyping or whole genome sequencing can happen to the testing, the

Competing interests: AMcN is the director of a pillar 2 testing laboratory based at University of Birmingham.

Commissioned, not peer reviewed.

This article is made freely available for use in accordance with BMJ's website terms and conditions for the duration of the covid-19 pandemic or until otherwise determined by BMJ. You may use, download and print the article for any lawful, quicker the data can be used and actioned. 
non-commercial purpose (including text and data mining) provided that all copyright notices and trade marks are retained. 